\title{
Capacity Retention Analysis in Aluminum-Sulfur Batteries
}

\author{
Jasmin Smajic, Shianlin Wee, Filipa R. Fernandes Simoes, Mohamed N. Hedhili, Nimer Wehbe, \\ Edy Abou-Hamad, and Pedro M. F. J. Costa*
}

Cite This: ACS Appl. Energy Mater. 2020, 3, 6805-6814

Read Online

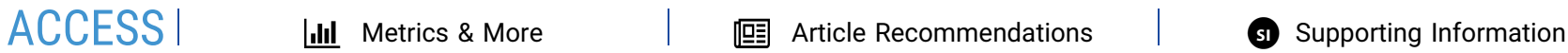

ABSTRACT: The electrochemical performance of aluminum-sulfur batteries is beset by poor stability and sluggish charge-storage properties. To address these issues, carbon allotropes have been used as electrode fillers, but successful outcomes remain inexplicably elusive. Here, a composite of sulfur and small-diameter single-walled carbon nanotubes was studied as a cathode for $\mathrm{AlCl}_{3}$ : [EMIM]-based aluminum batteries. The presence of carbon nanotubes, while enabling a high capacity $(1024$ $\mathrm{mAh}^{-1}$ ) with slower decay and reducing the electrolyte-to-sulfur ratio, is insufficient to fully stabilize the cell's performance. In fact, the main obstacle is in the interaction between sulfur and chloroaluminate ions. As we show, there is a gradual buildup of insoluble and poorly conductive

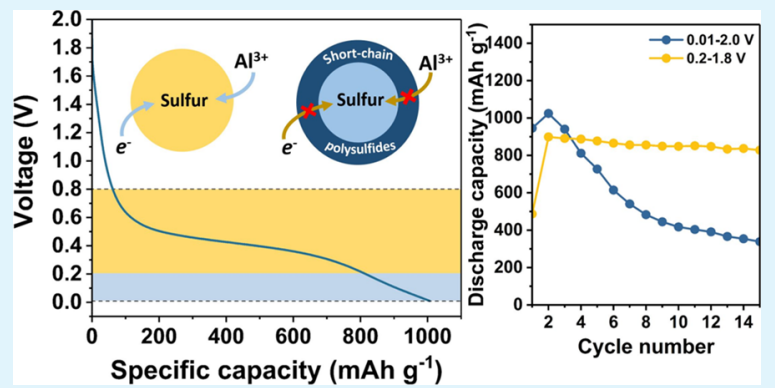
discharge products that inhibit the diffusion of electroactive ions and, ultimately, cause capacity decay. Overall, this work sheds light on the carbon-sulfur-electrolyte interactions and their role on the underlying charge-storage mechanism of aluminum-sulfur batteries.

KEYWORDS: aluminum, sulfur, carbon nanotubes, electrodes, batteries

\section{INTRODUCTION}

Lithium-ion batteries have conquered the portable electronics market. However, the push to electrify the transportation sector and the need to counteract the intermittency of renewable energy grids have laid bare the limits of this technology. ${ }^{1,2}$ Responding to the pressing demand for low-cost batteries with high energy density and being made out of abundant materials is, therefore, one of the primary interests in the energy storage research field. Recently, much attention has been given to alternative battery systems based on $\mathrm{Na}, \mathrm{K}, \mathrm{Mg}$, $\mathrm{Ca}$, and $\mathrm{Al}^{3-7}$ Of these, aluminum stands out as a particularly promising element. Besides being environmentally and toxicologically benign, it is the most abundant metal on earth, with an industrially mature approach for mineral extraction and processing. In addition, aluminum has a density of $2.7 \mathrm{~g} \mathrm{~cm}^{-3}$ and its ions are trivalent. ${ }^{8}$ These properties explain its remarkable theoretical gravimetric and volumetric capacities of $2980 \mathrm{mAh} \mathrm{g}^{-1}$ (second only to lithium) and 8046 $\mathrm{mAh} \mathrm{cm}^{-3}$ (the highest known for any battery electrode element), respectively. ${ }^{9,10}$

To fulfill its potential, Al battery systems will need to integrate an inexpensive and performant cathode. Graphitic cathodes have been successfully used in other battery chemistries, but their capacity in $\mathrm{Al}$ batteries is limited. ${ }^{11-13}$ Alternatively, sulfur could be an excellent electrode material. Besides a streamlined and geographically well-distributed production, this group 16 element can deliver considerably high theoretical capacities of $1672 \mathrm{mAh} \mathrm{g}^{-1}$ and $3340 \mathrm{mAh}$ $\mathrm{cm}^{-3}$ over a two-electron reduction process per atom. Since $S$ stores charge through a conversion reaction, the intercalation of bulky chloroaluminate ions (typically present in $\mathrm{Al}$ battery nonaqueous electrolytes) is avoided. ${ }^{14}$ Consequently, coupling an Al-based anode with a S-based cathode offers the promise of a low-cost battery with a remarkable theoretical energy density of $1371 \mathrm{Wh} \mathrm{kg}^{-1}$ (or $2742 \mathrm{Wh} \mathrm{L}^{-1}$; see the Supporting Information, Section S1.1).

The first report of a rechargeable Al-S battery dates back to 2016. ${ }^{15}$ Then, a $S$ cathode (integrating an activated carbon cloth that acted as the conductive matrix) was used in conjunction with an electrolyte of $\mathrm{AlCl}_{3}$ dissolved in 1-ethyl-3methylimidazolium chloride $([\mathrm{EMIm}] \mathrm{Cl})$. Interestingly, in the past 4 years, there were only few other reports describing the use of carbon-sulfur composite cathodes with the nonaqueous $\mathrm{AlCl}_{3}$ :[EMIM]Cl electrolyte. ${ }^{14,16-18}$ These studies generally make reference to a synergy between the carbon materials and sulfur, which improves the electrochemical performance of $\mathrm{Al}$ battery cathodes. Judging from the literature on the state of the art (Figure S1), it appears that this synergetic benefit applies solely to the initial cycles because the capacity decays rapidly. Observed also in other metal-S batteries, capacity fade has

Received: April 22, 2020

Accepted: June 15, 2020

Published: June 15, 2020 
a)

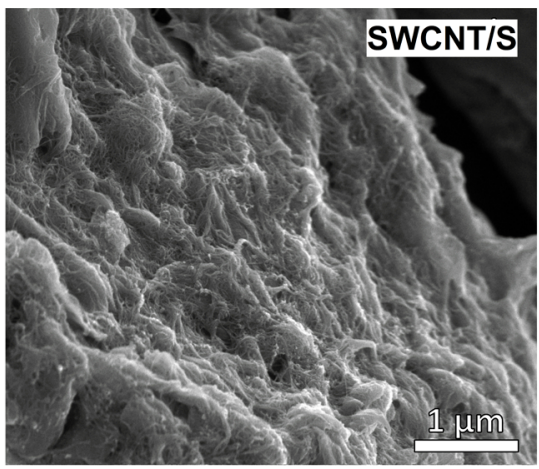

c)

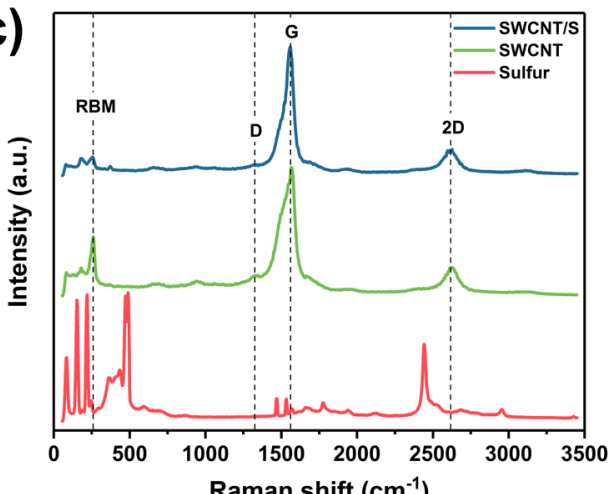

Raman shift $\left(\mathrm{cm}^{-1}\right)$

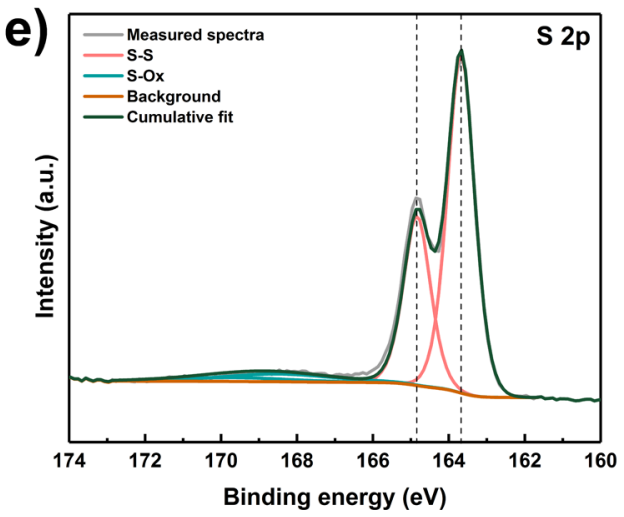

b)

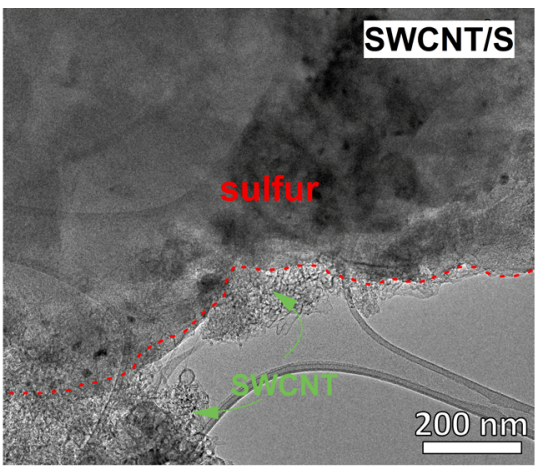

d)

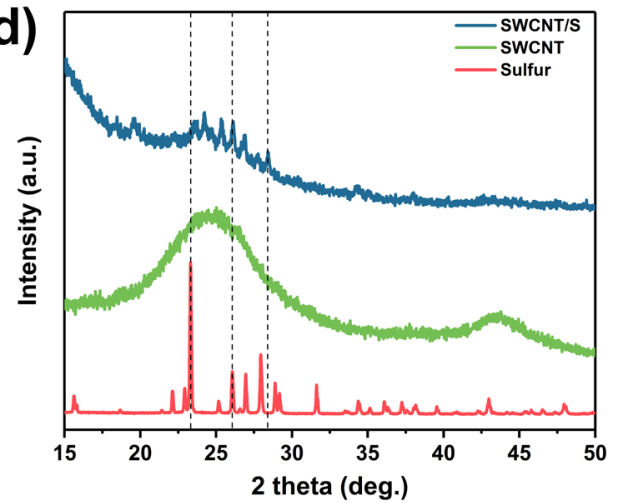

f)

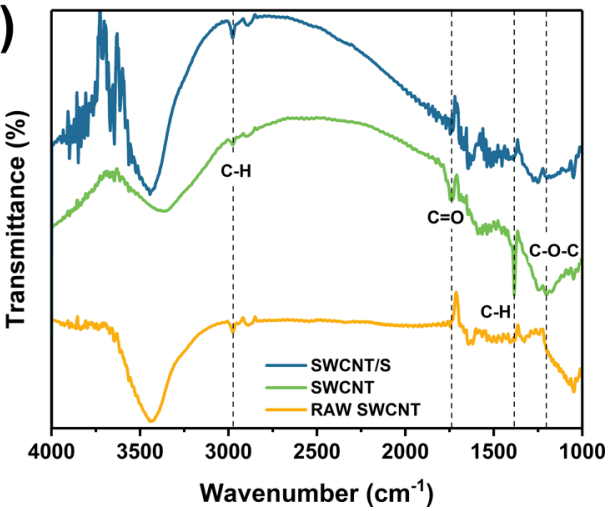

Figure 1. Materials characterization of SWCNT, S, and SWCNT/S materials: (a) SEM, (b) TEM, (c) Raman, (d) XRD, (e) S 2p XPS, and (f) FTIR spectra.

been attributed to a variety of reasons: from the insulating properties of S and/or its loss into the electrolyte (shuttling) to the kinetically limited solid-solid interconversion between $S$ and its products. ${ }^{14-16}$ In Al-S batteries, a complete understanding for the (invariably) rapid decay and, ultimately, the overall operation mechanism is still missing.

Herein, we resort to cathodes of small-diameter singlewalled carbon nanotubes (SWCNT) mixed with $S$ as a case study to explore the reasons for capacity loss in rechargeable, room-temperature Al-S batteries. Our study reveals that, upon cycling, due to the interaction between the electrolyte and sulfur, the electrodes are quickly deactivated by the buildup of a surface passivating layer composed of insoluble discharge products.

\section{EXPERIMENTAL SECTION}

2.1. Materials Synthesis. The SWCNT/S composite was synthesized through a nondestructive sublimation-deposition method (Figure S2). First, $100 \mathrm{mg}$ of SWCNT ( $\geq 77 \%$, Sigma-
Aldrich) was refluxed in $100 \mathrm{~mL}$ of concentrated nitric acid $\left(\mathrm{HNO}_{3}\right)$ for $3 \mathrm{~h}$ and at $100{ }^{\circ} \mathrm{C}$. After cooling down to room temperature, the nanotubes were thoroughly washed with deionized water (until $\mathrm{pH} \approx$ 7) and vacuum-filtered. The purified SWCNT were then demoisturized overnight at $60{ }^{\circ} \mathrm{C}$ and under vacuum. The dry SWCNT and $S$ powder (99.98\%, Sigma-Aldrich) were mixed by hand using an agate pestle and mortar and in equal proportions $(20 \mathrm{mg}$ total) for 15 min. Afterward, the black colored mixture was sealed in a quartz ampoule under vacuum $\left(<10^{-2} \mathrm{mbar}\right)$ and heated in a muffle furnace at $150{ }^{\circ} \mathrm{C}$ for $24 \mathrm{~h}$ (with a heating rate of $2{ }^{\circ} \mathrm{C} \mathrm{min}{ }^{-1}$ ). Finally, the ampoule was allowed to naturally cool down to room temperature and opened, and the powdered composite material was collected.

To prepare the electrolyte, 1-ethyl-3-methylimidazolium chloride ([EMIm] Cl) (98\%, Sigma-Aldrich) was first heated in an evacuated tube furnace at $70{ }^{\circ} \mathrm{C}$ and for $16 \mathrm{~h}$ to remove residual water. Avoiding exposure to air, the resulting powder was transferred to an Ar-filled glove box $\left(<0.5 \mathrm{ppm} \mathrm{O}_{2},<0.5 \mathrm{ppm} \mathrm{H}_{2} \mathrm{O}\right)$. The anhydrous $\mathrm{AlCl}_{3}$ powder (99.99\%, Alfa Aesar) was used as-received. The $\mathrm{AlCl}_{3}$ : [EMIm $] \mathrm{Cl}=1.3\left(\mathrm{~mol} \mathrm{~mol}^{-1}\right)$ electrolyte was prepared inside the glove box by slowly adding the $\mathrm{AlCl}_{3}$ to $[\mathrm{EMIm}] \mathrm{Cl}$. Mixing of the two powders produced a clear light-yellow liquid through an exothermic 
reaction. Finally, the obtained electrolyte was stirred for $30 \mathrm{~min}$ and left to stand.

2.2. Materials Characterization. The structural analysis was performed by powder X-ray diffraction (XRD) on a Bruker D8 ADVANCE and using $\mathrm{Cu} \mathrm{K} \alpha$ radiation $(\lambda=1.54 \AA)$ at a scan rate of $5^{\circ} \mathrm{min}^{-1}$ and increments of $0.02^{\circ}$. Raman spectra were recorded on a WITec alpha300 RA system with a $532 \mathrm{~nm}$ solid-state laser. Scanning electron microscopy (SEM) was carried out either on a FEI Nova Nano or a FEI Magellan, both operated at $5 \mathrm{kV}$. Transmission electron microscopy (TEM) was performed on a Thermo Fisher Scientific Titan CT, operated at $300 \mathrm{kV}$. Fourier transform infrared spectroscopy (FTIR) spectra were collected on a Thermo Scientific Nicolet iS10 spectrometer. Thermogravimetric analysis (TGA) was performed on a NETZSCH TG $209 \mathrm{~F} 1$ Libra in a $\mathrm{N}_{2}$ atmosphere with a flow of $20 \mathrm{~mL} \mathrm{~min}{ }^{-1}$, ranging from room temperature to 1000 ${ }^{\circ} \mathrm{C}\left(10{ }^{\circ} \mathrm{C} \mathrm{min}^{-1}\right)$. The X-ray photoelectron spectroscopy (XPS) characterization was performed on a Kratos Axis Supra instrument, equipped with a monochromatic $\mathrm{Al} \mathrm{K} \alpha \mathrm{X}$-ray source $(h \nu=1486.6$ $\mathrm{eV}$ ) and operated at a power of $150 \mathrm{~W}$ under ultrahigh-vacuum conditions $\left(10^{-9} \mathrm{mbar}\right)$. The $1 \mathrm{D}{ }^{13} \mathrm{C}$ magic angle spinning (MAS) nuclear magnetic resonance (NMR) data was recorded on a Bruker AVANCE III spectrometer, operated at resonance frequencies of 600 $\mathrm{MHz}$ and using a conventional double-resonance $3.2 \mathrm{~mm}$ crosspolarization (CP) MAS probe. Inductively coupled plasma-optical emission spectrometry (ICP-OES) was done on an Agilent 5110 bearing a synchronous vertical dual-view configuration at wavelengths between 167 and $780 \mathrm{~nm}$. The samples were prepared using a microwave-assisted acid digestion protocol detailed elsewhere. ${ }^{19}$

2.3. Electrochemical Measurements. The electrochemical measurements were taken on a Bio-Logic VMP3 potentiostat in a Swagelok two-electrode cell configuration. The cells were assembled inside an Ar-filled glove box (LABstar, MBraun) using S, SWCNT, or SWCNT/S composite as working electrodes and an Al foil (99.999\%, $0.25 \mathrm{~mm}$, Sigma-Aldrich) as a counter/reference electrode. The cathode was fabricated by mixing 50 wt $\%$ active material (SWCNT or S), 30 wt \% acetylene carbon black (CB), and 20 wt \% polyvinylidene fluoride (PVDF) with $N$-methyl-2-pyrrolidone (NMP). The binder PVDF was selected owing to its good synergy with carbonaceous materials and limited adversity. ${ }^{20}$ The obtained slurry was transferred into a vial and homogenized by magnetic stirring (for $24 \mathrm{~h}$ ). The slurry was then spread on a niobium foil $(0.25 \mathrm{~mm}$ thickness, $19 \mathrm{~mm}$ diameter, Sigma-Aldrich) and dried overnight at $100{ }^{\circ} \mathrm{C}$ and under an inert atmosphere. Borosilicate microfiber filters (grade $\mathrm{GF} / \mathrm{F}$, Whatman), soaked with the $\mathrm{AlCl}_{3}:[\mathrm{EMIm}] \mathrm{Cl}=1.3\left(\mathrm{~mol} \mathrm{~mol}{ }^{-1}\right)$ electrolyte, were used as separators in the electrochemical cells. These filters were desiccated at $150^{\circ} \mathrm{C}$, in vacuo $(10 \mathrm{mbar})$ and for $16 \mathrm{~h}$, in a tube furnace and subsequently introduced into the Ar-filled glove box, avoiding air exposure. Additionally, all cell components were dried inside the glove box on a hotplate at $150{ }^{\circ} \mathrm{C}$ and overnight. Electrochemical impedance spectroscopy (EIS) was carried out from $10 \mathrm{mHz}$ to $100 \mathrm{kHz}$ with an amplitude of $5 \mathrm{mV}$. Before each galvanostatic experiment, the cell was left to stand for $12 \mathrm{~h}$ (in open circuit conditions) to properly wet the electrodes with the electrolyte. Afterward, the cells were subjected to an activation step with cyclic voltammetry $(\mathrm{CV})$, employing 10 cycles at $1 \mathrm{mV} \mathrm{s}^{-1}(0.01-2.0 \mathrm{~V})$. All electrochemical measurements were carried out at room temperature. All capacities are reported per mass of the active material.

\section{RESULTS AND DISCUSSION}

With a surface area of $\sim 600 \mathrm{~m}^{2} \mathrm{~g}^{-1}$, SWCNT should accommodate well the active material of Al-S battery cathodes, which is sulfur (Figure S1b). ${ }^{21}$ To test this, the as-received carbon nanotubes were first purified, ensuring that their structural order remained intact (Figure S3). This was done to remove the electrochemical interference from growth catalyst remainders as these will affect the performance of the batteries (Figure S1c). Next, the total amount of S present in the
SWCNT/S powder was established by TGA ( 35 wt \%) (Figure S4). This composite was further analyzed to understand the interaction between its two components. From the SEM (Figure $1 \mathrm{a}$ and Figure S5a,b) and TEM (Figure $1 \mathrm{~b}$ and Figure $\mathrm{S} 5 \mathrm{c}, \mathrm{d}$ ), it was possible to infer the degree of mixing $\mathrm{C}$ and $\mathrm{S}$ as well as the microscaled morphology of the powder. Single-component aggregates were mostly absent, thereby attesting to a successful dispersion. Following this and to gain insight on the averaged structural characteristics of the composite, Raman and powder XRD were used. The Raman spectrum of $S$, as shown in Figure 1c, contains a series of prominent peaks in the lower frequency region with the dominant ones resonating at 152, 219, and 480 $\mathrm{cm}^{-1}$. As per the literature, these are typical of the orthorhombic polymorph of $S$, known to be stable at room temperature. ${ }^{22}$ Also, in Figure 1c, the spectrum of the purified SWCNT shows the expected radial breathing mode (RBM) peaks as well as the higher-frequency $\mathrm{D}, \mathrm{G}$, and $2 \mathrm{D}$ bands. These are all visible in the composite, too. The RBM region has a dominant peak at $260 \mathrm{~cm}^{-1}$, which correlates with a nanotube diameter of $\sim 1 \mathrm{~nm}$ (see the Supporting Information, Section S1.2). In the context of a battery, a small diameter is advantageous as the nanotubes are more resistant to radial stress and have a smaller Poisson's ratio. These characteristics help preserve the structural integrity of the SWCNT when they are subjected to pressure such as that exerted by volume changes resulting from the battery operation mechanism. ${ }^{23}$ The $D$ and $G$ bands are present at $\sim 1330$ and $1565 \mathrm{~cm}^{-1}$, respectively. Since the $\mathrm{D}$ band originates from the cumulative breaking of symmetry (due to defects, edges, etc.), its low intensity is indicative of the high structural quality of the nanotubes. This is corroborated by the low value of the $\mathrm{D}$ - to G-band ratio $(\sim 0.1)$. As for the $G$ band, its asymmetric shape confirms the single-walled nature of the nanotubes. ${ }^{24}$ Interestingly, the SWCNT/S spectrum is similar to that of the SWCNT with one notable difference being the intensity reduction of the main RBM peak. It is possible that the dampening of the radial vibrations occurred due to $S$ coating (or filling) of the nanotubes. In the composite spectrum, the peaks of $S$ are entirely absent (Figure 1c). Likely, this is because of the well-dispersed nature of the $S$ in addition to the stronger light absorption coefficient of the black carbonaceous material. The signals of the latter will greatly outweigh those of $\mathrm{S.}^{25}$

In Figure 1d, the XRD diffractogram of the $S$ powder confirms the Raman analysis. The pattern has a series of sharp peaks, which were assigned to the crystalline orthorhombic phase (JCPDS card no. 08-0247). In the same figure, the SWCNT presence is asserted by the two broad peaks at $25^{\circ}$ and $44^{\circ}$, typical of these carbon allotropes. As for the diffractogram of the SWCNT/S composite, this seems to be a sum of the two components. Still, a slight upshift of the peak positions $\left(\sim 0.2^{\circ}\right)$ is observed and attributed to the accumulated stresses in $S$ (due to exposure to heat during the composite fabrication). There is also an interesting change in the relative intensities of the S peaks; whereas the (222) peak, at $23.3^{\circ}$, is dominant in the $S$ pattern, its intensity is drastically reduced in the SWCNT/S product. This is a possible consequence of morphological and/or structural rearrangements such as the preferential deposition of $S$ species on the surface of the anisotropic carbon nanotubes. To clarify, the XRD diffractograms of the $S$ component and the composite were compared to a physical mixture of SWCNT 

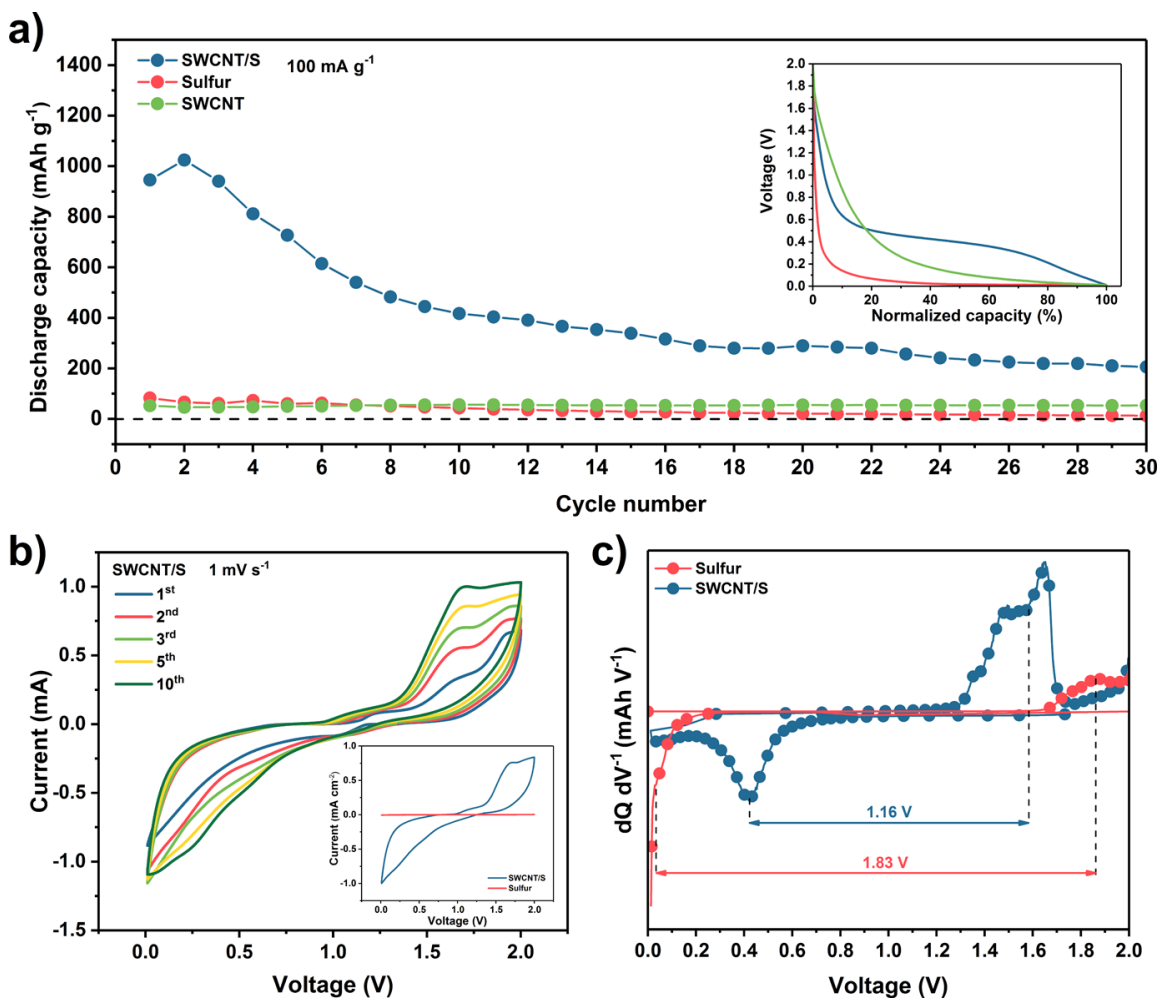

Figure 2. Electrochemical performance of SWCNT, S, and SWCNT/S materials: (a) cycling stability at a current density of $100 \mathrm{~mA} \mathrm{~g}^{-1}$ (inset: voltage profiles of the composite and constituent materials), (b) cycling voltammogram at a voltage sweep of $1 \mathrm{mV} \mathrm{s}^{-1}$, and (c) differential capacity plot.

and $S$ that was not subjected to heat treatment (Figure S6). Interestingly, the orthorhombic (222) S peak remains dominant in the nonheated mixture. Thus, the pattern variation of the composite and mixture can be interpreted as the result of a phase transformation at higher temperatures, such as the orthorhombic to the monoclinic one. ${ }^{26}$ Knowing that the crystal structure of materials depends heavily on their heat treatment (especially on the cooling rate), and considering that the SWCNT have an extremely high thermal conductivity $\left(\sim 3 \times 10^{3} \mathrm{~W} \mathrm{mK}^{-1}\right)$, it is plausible that the nanotubes acted as a heat sink..$^{27,28}$ When the heat treatment was applied, the rapid sulfur-to-nanotube heat transfer created a quenching effect, which kinetically impaired the full reconversion of $S$ from the monoclinic to the orthorhombic phase. Potentially, the structural diversity of $S$ could be used to increase the volumetric energy density of these batteries $(2.18$ $\mathrm{g} \mathrm{cm}^{-3}$ for monoclinic vs $2.07 \mathrm{~g} \mathrm{~cm}^{-3}$ for orthorhombic). ${ }^{29}$

After assessing the morphology and structure of the SWCNT/S composite, XPS was conducted to identify possible chemical interactions between the $S$ and SWCNT. The highresolution S 2p XPS spectrum, as shown in Figure 1e, exhibits a clear splitting of the $S$ spin-orbit. The broad and lowintensity peaks, between 167 and $170 \mathrm{eV}$, are attributed to various sulfate $\mathrm{S}-\mathrm{O}_{x}$ bonds. ${ }^{15}$ Further to this, the $\mathrm{C} 1 \mathrm{~s}$ and $\mathrm{O}$ 1s XPS spectra (Figure S7) show typical $\mathrm{C}=\mathrm{C} / \mathrm{C}-\mathrm{C}$ peaks along with the presence of various oxygen-containing functional groups. This analysis was supplemented with FTIR spectroscopy (Figure 1f) where the peaks corresponding to the oxygen-containing functional groups, also present in the purified SWCNT, got reduced in intensity after the heat treatment with $\mathrm{S}$. To confirm the integrity of the $\mathrm{sp}^{2}$-C lattice and the (almost exclusively) physical interaction between the two components in the SWCNT/S composite, solid-state ${ }^{13} \mathrm{C}$ NMR spectra were acquired (Figure $\mathrm{S} 8$ ). The $\mathrm{C}=\mathrm{C}$ peak of the SWCNT (117.4 ppm) was seen to enlarge and shift to $121.6 \mathrm{ppm}$. According to the literature, these changes are expected in the added presence of electronegative elements such as $\mathrm{S} .{ }^{30}$ Overall, the set of spectral analyses is coherent and informs that the heat treatment did not lead to considerable chemical interactions between $\mathrm{S}$ and $\mathrm{C}$ species. ${ }^{31}$

After asserting that the heated SWCNT/S powder was a physical mixture of the two components (albeit with a morphological and structurally modified $S$ ), the cathodes for the Al-S battery were assembled (SWCNT, S, and SWCNT/ $S)$. All sulfur cells showed an open circuit potential (OCV) of around $1.2 \mathrm{~V}$. This is close to the theoretical electromotive force of $1.28 \mathrm{~V}$ for an $\mathrm{Al}-\mathrm{S}$ cell (Supporting Information, Section S1.1) and confirms a correct assembly of the cells. Owing to its electrochemical inertness (Figure S9a) and greater abundance than other transition metals (Mo, Ta, and $\mathrm{W})$ commonly used as current collectors in Al batteries, $\mathrm{Nb}$ was selected as the current collector. ${ }^{32}$ PVDF and carbon black, which also integrated the slurry, showed negligible contributions to the overall capacity $\left(<5 \mathrm{mAh} \mathrm{g}^{-1}\right)$ (Figure S9b). ${ }^{20}$

The main electrochemical characteristics and performance of the assembled batteries are presented in Figure 2. Figure 2a shows the cycling stability and voltage profiles (Figure S10, inset) of the SWCNT, the S, and the SWCNT/S composite (at $\left.100 \mathrm{~mA} \mathrm{~g}^{-1}\right)$. The two components, SWCNT and S, have second discharge capacities of 46 and $66 \mathrm{mAh} \mathrm{g}^{-1}$, respectively. The low $S$ capacity is surprising and indicative of poor utilization of the active material (only $4 \%$ of the available mass). Possibly, only the surface of $S$ participated in the 
a)

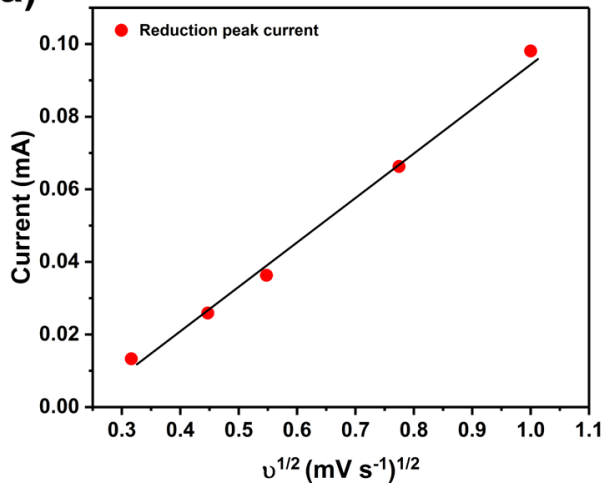

b)

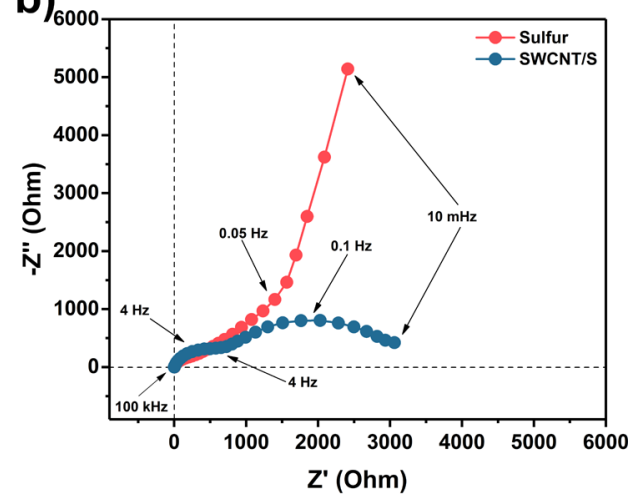

Figure 3. (a) Randles-Sevcik plot of the cathodic peak current for the SWCNT/S cathode. (b) EIS Nyquist spectrum of S and SWCNT/S.

electrochemical reactions. The SWCNT/S composite, on the other hand, showed a remarkable discharge capacity of 1024 $\mathrm{mAh} \mathrm{g}^{-1}$ (at the second cycle). This corresponds to a $\mathrm{S}$ utilization of $61 \%$ and represents a stark improvement over the single-component cathodes. The Coulombic efficiency rapidly approaches $80 \%$ (Figure S11). Clearly, in addition to being a good physical support to accommodate the active material, the SWCNT network acts as a conductive pathway that promotes access of the electrolyte to the redox sites of $S$ (justifying its much higher utilization rate). This is also a logical consequence of the increased contact area between the electrolyte and S. Graphitic carbons, especially small diameter nanotubes, are known to have excellent wettability in ionic liquids. $^{33-35}$ Here, this behavior was confirmed by the low electrolyte-to-sulfur (E/S) ratio achieved $\left(17 \mu \mathrm{L} \mathrm{mg}^{-1}\right)$. When compared to the previous literature, this value corresponds to a three-fold reduction in the electrolyte amount while obtaining a similar electrochemical performance (Figure S1d). Note however that, while a high $\mathrm{E} / \mathrm{S}$ ratio may allow better electrochemical performance (i.e., capacity, cycle number, $S$ utilization, etc.), it is unsuitable for practical applications as it decreases the overall cell energy density. ${ }^{36}$

Even though the capacity of the SWCNT/S fades with cycling (Figure 2a and Figure S12a), the presence of the nanotubes slightly slows down the steep decay rates seen in the $\mathrm{S}$ cathodes (Figure S13a) and in previous reports of $\mathrm{Al}-\mathrm{S}$ batteries (despite using at least 3 times less electrolyte; Figure S1a, S1b, and S1d). This behavior was maintained even when the composite cell was ran at a higher current density (Figure $\mathrm{S} 12 \mathrm{~b})$. The cyclic voltammogram (CV) of the SWCNT/S (Figure 2b) exhibits wide cathodic and anodic peaks in the $0.6-0.2$ and $1.3-1.7 \mathrm{~V}$ range, respectively. The intensity of these reversible peaks increases during the initial cycles, indicating a progressive electrochemical activation of the cathode. The CV of $S$, on the other hand, shows incomplete peaks at $\sim 0.05$ and $\sim 1.9 \mathrm{~V}$, indicating high cell polarization (Figure S13b). When the differential capacity profile for this cell is plotted alongside that of the composite, the change in polarization is $\sim 0.7 \mathrm{~V}$ (Figure $2 c$; note that the differential plot of carbon black does not exhibit peaks; Figure S14). Therefore, the presence of the SWCNT effectively reduces the cell polarization in Al-S batteries by $\sim 37 \%$. Overall, the present system confirms the beneficial role of small-diameter SWCNT in improving the utilization, reversibility, and $\mathrm{E} / \mathrm{S}$ ratio of $\mathrm{S}$ based cathodes.

The behavior of the SWCNT/S cathode is consistent with the results described by other groups who used different carbon materials and different amounts of electrolytes. ${ }^{15,16,37}$ From this, the main hurdle in Al-S batteries appears to be not in the cathode design or which carbon is used but in the interaction of the electrolyte and S. Low capacity retention has been understood as a consequence of the solid-solid electrochemical interconversion between $S$ and its products (both electrical insulators), phenomena that kinetically limit Al-S batteries. ${ }^{15,16}$ Other suspected limiting factors are the dissociation of $\mathrm{Al}_{2} \mathrm{Cl}_{7}^{-}$, sluggish charge transfer, shuttling of polysuflides, and side reactions such as the nucleophilic attack of $\mathrm{S}$ by $\mathrm{EMI}^{+}$ions. ${ }^{37} \mathrm{~A}$ battery chemistry as complex as the Al$S$ one is likely to have a number of different interplaying phenomena that result in high capacity fades. Nevertheless, it should be possible to identify those majorly responsible for it.

Our data implies that the interaction between chloroaluminate ions and sulfur is diffusion-limited. This is clearly evidenced by the linear dependence of the peak current to the square root of the voltage sweep rate in the RandlesSevcik plot (Figure 3a).

To better understand this diffusion, we studied the Nyquist plots of SWCNT/S and S under open circuit conditions $(\mathrm{OCV})$, as shown in Figure $3 \mathrm{~b}$ (the equivalent circuit fittings are shown in Figures S15 and S16). The most notable difference is in the low-frequency region, implying different mass diffusion regimes. In both cases, there is a strong deviation from the Warburg semi-infinite diffusion regime, suggesting obstacles to the progress of the chloroaluminate ions into the cathode. While the Nyquist plot of S approaches a finite-space Warburg (FSW) diffusion, the one of SWCNT/S is characterized by a semicircle at lower frequencies, typical of finite-length Warburg (FLW) diffusion (see the Supporting Information, Section S1.3). Likely, the former is a consequence of the sluggish diffusion of chloroaluminate ions in S. As the anions cannot easily diffuse through the monolithic $S$, a gradual accumulation of charged species will occur at the electrode's surface. This will result in a blocking behavior, limiting Faradaic reactions and leaving the bulk of $S$ inactive. The semicircle, on the other hand, can be understood as diffusion across the entire $S$ layer up to an adsorbing boundary. Here, this boundary consists of the carbon nanotubes. Considering that, in the composite, part of the $S$ is arranged as a thin layer that coats the charge-conducting filler, shorter ion conduction paths are expected. This will significantly reduce the amount of inactive $S$ in the electrode as confirmed by the difference in $S$ utilization in the absence or presence of SWCNT (4\% vs $61 \%$, respectively). Hence, the role of the SWCNT is three-fold: (i) it acts as a substrate for the deposition of a thin layer of the 


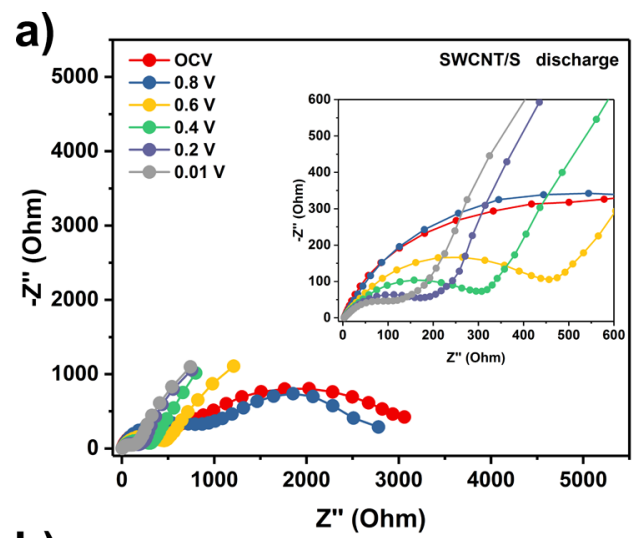

c)
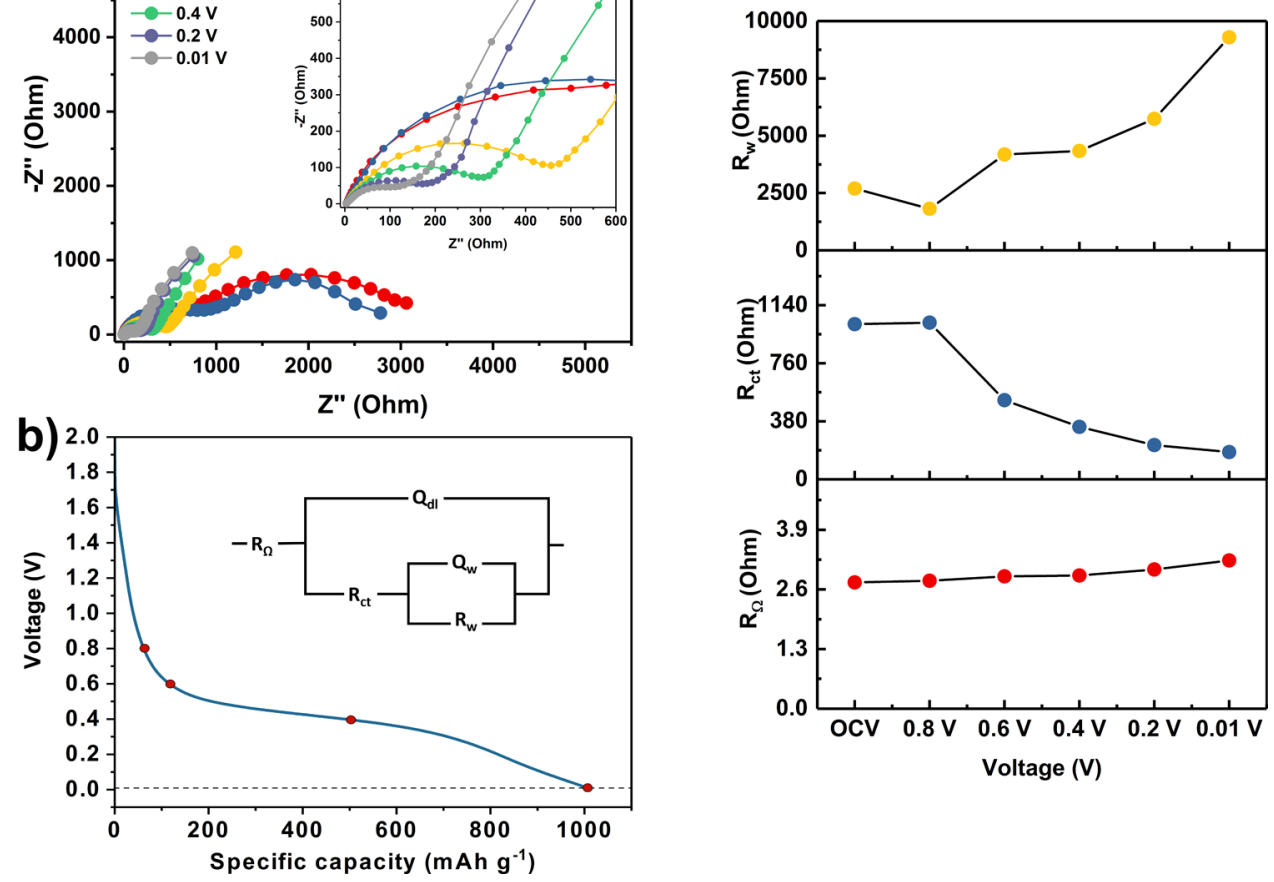

Figure 4. EIS of SWCNT/S: (a) Nyquist plots as a function of the depth of discharge, (b) discharge points at which EIS was measuredwith the equivalent circuit (inset), and (c) resistance values as a function of the depth of discharge.

active material; (ii) it acts as a "charge" buffer by redistributing excess localized carriers and electrically homogenizing the interphase between the solid and liquid; and (iii) it stabilizes, mechanically, the active material against pulverization.

To clarify the capacity decay, the EIS of the SWCNT/S was studied as a function of discharge voltage (Figure 4). Generally, a Nyquist plot is characterized by high-, medium-, and low-frequency regions. The high-frequency intersect with the $x$ axis is related to the ohmic resistance and includes the contributions from the conductivity of the electrolyte as well as the contact resistances (which result from the cell setup and current collectors). Any semicircles in the high- or mediumfrequency regions are associated with interfacial phenomena and, as such, are under kinetic control. The low-frequency region is attributed to mass diffusion-controlled phenomena such as the diffusion of electroactive ions in the electrolyte or the active material. These physical phenomena ought to be considered when choosing an equivalent electrical circuit to model the impedance data. A good fit between experimental and simulated circuits is not sufficient, and it should always be coupled with the physical explanation for each of the elements on the circuit. Here, we fit all obtained spectra with the equivalent circuit shown in Figure $4 \mathrm{~b}$ (inset). A constant phase element ( $Q$ ) is used, instead of a capacitor, to compensate for the nonideal state of the electrode such as its roughness and porosity. In the low-frequency region, a similar approach is used due to considerable deviations from the Warburg semiinfinite diffusion regime. ${ }^{38}$ Resistance originating from the aluminum anode is known to be negligible, so we assume that the obtained values are, predominantly, from the processes occurring in the cathode. ${ }^{15}$ For more details, the EIS fittings for the SWCNT/S cathode are shown in Figure S15.

Following the above, the discharge-dependent EIS spectra of the SWCNT/S (Figure 4a) are characterized by a semicircle in the high-frequency region and different types of low-frequency responses. The high-frequency semicircle's intercept with the real impedance axis is attributed to the ohmic resistance $\left(R_{\Omega}\right)$ and was seen to slightly increase with deeper discharge (Figure $4 c$, bottom plot). Its diameter is attributed to the charge transfer resistance $\left(R_{\mathrm{ct}}\right)$ and was seen to sharply decrease at voltages lower than $0.8 \mathrm{~V}$ (Figure $4 \mathrm{c}$, middle plot). This is likely a consequence of the consumption of elemental $S$ through electrochemical reactions, which are initiated at this voltage threshold. The subsequent dissolution of the products (i.e., high to intermediate order polysulfides) into the electrolyte would facilitate the electronic contact between particles. ${ }^{39}$ This is typically related to increased viscosity of the electrolyte, which causes an increase in $R_{\Omega}{ }^{16,39}$ The lowfrequency region showed a strong deviation from the semiinfinite linear diffusion. Furthermore, it was seen that its resistance $\left(R_{\mathrm{w}}\right)$ rapidly increases at discharge voltages lower than $0.8 \mathrm{~V}$ (Figure 4c, top plot), which is indicative of a progressively more difficult diffusion as sulfur gets reduced further to lower-order polysulfides. This implies that the discharge products are the reason behind the surge in resistance.

Following the discharge depth analysis, and to shed light on the interaction between the electrolyte and S, the EIS spectra of the $S$ electrode were collected as a function of the cycle number (Figure 5a,b and Figure S16). Again, the $R_{\Omega}$ shows a slight increase, implying a continual loss of $S$ products into the electrolyte, presumably through the dissolution of high to intermediate polysulfides (Figure 5a, bottom plot). ${ }^{16}$ Both the charge-transfer $\left(R_{\mathrm{ct}}\right)$ and diffusion $\left(R_{\mathrm{w}}\right)$ resistances increase considerably with cycling (Figure 5a, middle and top plot, respectively), indicative of a progressive degradation of the electrode. The consequence is charge-transfer impairment along with a gradually more inhibited ion diffusion. If the 
a)

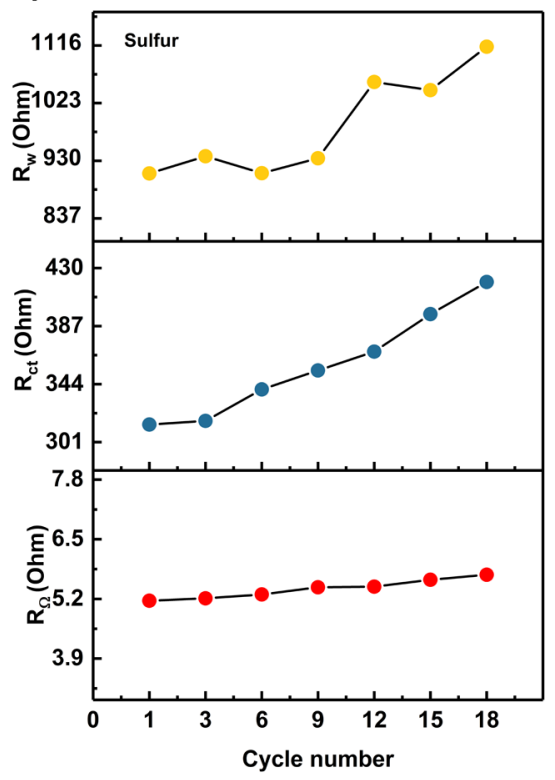

b)



c)

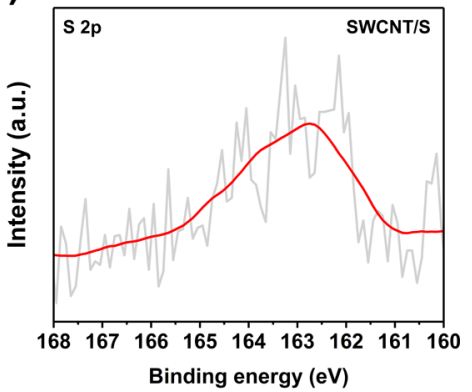

d)

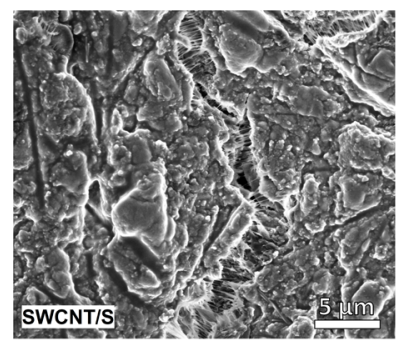

e)

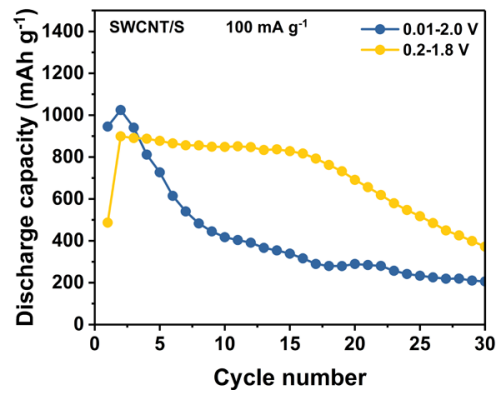

Figure 5. (a) EIS resistance values and (b) EIS Nyquist plots for a $S$ cathode as a function of the cycle number (inset: high-frequency region Nyquist plots). (c) S 2p XPS spectrum of a fully discharged sulfur cathode. (d) Postmortem SEM image of the SWCNT/S cathode surface. (e) Comparison of SWCNT/S cathode cycling stability with different voltage limits.

reason for this is the discharge products, as evidenced above, then it would seem that they cannot be fully reoxidized. This not only causes an inefficient $S$ utilization but also causes their gradual buildup on the electrode's surface and limits the access of chloroaluminate ions to redox active sites, ultimately deactivating the electrode. The shortening, and eventual disappearance, of the plateau at $\sim 0.5 \mathrm{~V}$ added to the increase in polarization with cycling (Figure S10) and the continuous capacity fade; all corroborate the hypothesis of a passivating layer composed of insoluble discharge products on the sulfurbased cathodes.

To support our claim of irreversible formation of the discharge products, we did a postmortem analysis of the cycled SWCNT/S electrode and compared it to its pristine state. Indeed, the SEM of the cycled electrode shows a thick coating on its surface (Figure 5d and Figure S17). This passivating layer can be recognized when looking at the images of the surface in the initial state wherein the ropes of nanotubes are notoriously visible and ubiquitous (Figure 1a and Figure $\mathrm{S} 5 \mathrm{a}, \mathrm{b})$. To understand the chemical nature of this coating, the morphology study was followed by a spectroscopic analysis. The XPS S $2 p$ spectrum in Figure $5 c$ is characterized by a broad peak that is shifted to lower binding energies (cf. Figure 1e). Its width indicates a mixture of different oxidation states, which confirms the poor reversibility of the interconversion between elemental $S$ and its products. ${ }^{40}$ Considering that the peak maxima is close to $162.7 \mathrm{eV}$, it is likely that the majority of the products contain the partially $\left(\mathrm{S}_{x}^{-}\right)$or fully $\left(\mathrm{S}^{2-}\right)$ reduced species. The XPS $\mathrm{Al} 2 \mathrm{p}$ spectrum consists of only one peak centered at $74.3 \mathrm{eV}$ (Figure S18b), a position commonly attributed to the $3+$ oxidation state. Therefore, it is safe to assume that the reduction products are related to $\mathrm{Al}_{2} \mathrm{~S}_{3}$ with sulfur being in either $S_{x}{ }^{-}$or $S^{2-}$ states. ${ }^{15,41}$ In fact, from the XRD diffractograms (Figure S19), the disappearance of the $S$ peaks with cycling is obvious, indicating the complete reduction of the majority of elemental sulfur. Hence, the interconversion between different $S$ products seems to be poorly reversible and is in agreement with previous findings. ${ }^{15,16}$ If that is the case, then limiting the voltage window should improve the reversibility of the Al-S cell because the formation of passivating layers would be slowed down. Indeed, as seen in Figure 5e, when the voltage window is limited between 0.2 and $1.8 \mathrm{~V}$ (instead of 0.01 and $2.0 \mathrm{~V}$ ), the steep capacity decay was mitigated for a short number of cycles without significantly lowering the capacity.

All things considered, our findings show that the capacity decay is, in major part, due to the formation of dense and poorly conductive discharge products, which not only are difficult to reoxidize but most importantly also increase the resistance to ion diffusion. This results in a gradual increase of 
cell polarization and progressively limits the $S$ that can be accessed, ultimately causing the complete deactivation of the $S$ electrode. ${ }^{42}$ The consequence of this is shortening of the voltage profile plateaus (Figure S10) and steep capacity decay (Figure S12a). Of note, a similar phenomenon was previously reported for Li-S battery systems. ${ }^{39,43}$ In such cases, the capacity loss was generally attributed to the dissolution of the polysulfides (i.e., shuttle effect) or S into the electrolyte and to the irreversible formation of insoluble and insulating precipitates. This line of thought was analogously transferred to the field of Al-S batteries, resulting in works that try to improve the electrochemical performance by limiting polysulfide shuttling. ${ }^{17}$ In $\mathrm{Al}-\mathrm{S}$ batteries with the $\mathrm{AlCl}_{3}:[\mathrm{EMIm}] \mathrm{Cl}$ electrolyte, the case appears to be slightly different, considering that $S$ has very low solubility in this ionic liquid. As mentioned above, leaching of $S$ into the electrolyte is unlikely to be the critical reason for capacity fade. ${ }^{15}$ This suggestion is corroborated by the ICP-OES and optical (Figure S20) analysis of the cycled separators. After long-term cycling, only $376 \mathrm{ppm} \mathrm{S}$ species was found present in the SWCNT/S separator compared to the $418 \mathrm{ppm}$ measured from the $S$ cathode. Therefore, the loss of $S$ into the electrolyte is small and lessens slightly when carbon additives that can trap polysulfides, such as SWCNT, are added. Notwithstanding, this does not explain the large difference in the electrochemical behavior of the two cathodes.

In closure, the Al-S battery discharge proceeds through the continuous reduction of $\mathrm{S}$ to $\mathrm{Al}_{2} \mathrm{~S}_{3}$-like products via a series of polysulfide intermediate species. The redox process can be summarized by the following equations on the anode (eq. 1) and the cathode (eq. 2)

$$
\begin{aligned}
& 2 \mathrm{Al}+14 \mathrm{AlCl}_{4}^{-} \rightarrow 8 \mathrm{Al}_{2} \mathrm{Cl}_{7}^{-}+6 \mathrm{e}^{-} \\
& 8 \mathrm{Al}_{2} \mathrm{Cl}_{7}^{-}+6 \mathrm{e}^{-}+3 \mathrm{~S} \rightarrow \mathrm{Al}_{2} \mathrm{~S}_{3}+14 \mathrm{AlCl}_{4}^{-}
\end{aligned}
$$

It is clear that the interaction between $\mathrm{Al}$ and $\mathrm{S}$ is sluggish with a high kinetic barrier and that it requires the breaking of chloroaluminate ion bonds to free the aluminum ion following the sequence ${ }^{37}$

$$
\mathrm{Al}_{2} \mathrm{Cl}_{7}^{-} \rightarrow \mathrm{AlCl}_{3}+\mathrm{AlCl}_{4}^{-} \rightarrow 4 \mathrm{AlCl}_{4}^{-}+\mathrm{Al}^{3+}
$$

Moreover, the interconversion between elemental $S$ and $\mathrm{Al}_{2} \mathrm{~S}_{3}$ is not efficient, causing a gradual buildup of insoluble and poorly conductive precipitates that hinder the diffusion of charged species. ${ }^{16}$

From the above, the cathode deactivation can be mitigated, but not resolved, by the synergy of sulfur and carbonaceous materials such as SWCNT since this will help with the following:

- Dispersing $S$ as a thin layer coating the conductive substrate, thereby providing a more extended interface between the electrolyte and the active material as well as shorter charge diffusion paths.

- Limiting the loss of $S$, or its polysulfides, into the electrolyte due to a mesh-like barrier effect that results, in the present case, from the entanglement of the carbon nanotubes.

- Easier wetting of the electrode as this will allow a substantial reduction of electrolyte consumption, a point explained by the excellent affinity between graphitic materials (especially small diameter SWCNT) and electrolytes based on ionic liquids.
While a judicious design of the cathode can reduce the capacity decay, it is not a solution for these systems where the main hurdle is the interaction between $\mathrm{S}$ and the $\mathrm{AlCl}_{3}$ : [EMIM]Cl, the most popular electrolyte in the Al-S battery literature. As such, new electrolytes are urgently needed and should be the focus of future research in $\mathrm{Al}$ batteries.

\section{CONCLUSIONS}

A composite of sulfur and small diameter single-walled carbon nanotubes was synthesized and used as a cathode for nonaqueous reversible aluminum-sulfur batteries. The assembled electrode delivered a high capacity of $1024 \mathrm{mAh}$ $\mathrm{g}^{-1}$ and effectively reduced the cell polarization by $37 \%$. Moreover, the use of small-diameter SWCNT helped minimize the electrolyte-to-sulfur ratio down to $17 \mu \mathrm{g} \mathrm{ml}^{-1}$, a three-fold reduction compared to similar literature, an important step toward lean electrolyte conditions. Despite that, the capacity fade of the Al-S battery could not be fully reversed. A detailed study revealed that the consequence of the interaction between the electrolyte and $S$ is the buildup of insoluble and poorly conductive discharge products, which inhibit charge diffusion and progressively deactivate the electrode. This leads to the capacity fade. Overall, this works clarifies the carbon-sulfurelectrolyte interactions and their role in the underlying chargestorage mechanism of Al-S batteries.

\section{ASSOCIATED CONTENT}

\section{SI Supporting Information}

The Supporting Information is available free of charge at https://pubs.acs.org/doi/10.1021/acsaem.0c00921.

Mathematical calculations, literature review, and additional experimental results (PDF)

\section{AUTHOR INFORMATION}

\section{Corresponding Author}

Pedro M. F. J. Costa - Physical Science and Engineering Division, King Abdullah University of Science and Technology,

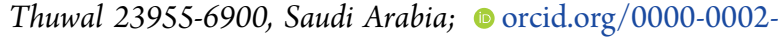
1993-6701; Email: pedro.dacosta@kaust.edu.sa

\section{Authors}

Jasmin Smajic - Physical Science and Engineering Division, King Abdullah University of Science and Technology, Thuwal 239556900, Saudi Arabia; 이이. orcid.org/0000-0003-2374-0901

Shianlin Wee - Physical Science and Engineering Division, King Abdullah University of Science and Technology, Thuwal 239556900, Saudi Arabia

Filipa R. Fernandes Simoes - Physical Science and Engineering Division, King Abdullah University of Science and Technology, Thuwal 23955-6900, Saudi Arabia

Mohamed N. Hedhili - Core Labs, King Abdullah University of Science and Technology, Thuwal 23955-6900, Saudi Arabia

Nimer Wehbe - Core Labs, King Abdullah University of Science and Technology, Thuwal 23955-6900, Saudi Arabia

Edy Abou-Hamad - Core Labs, King Abdullah University of Science and Technology, Thuwal 23955-6900, Saudi Arabia

Complete contact information is available at:

https://pubs.acs.org/10.1021/acsaem.0c00921

\section{Notes}

The authors declare no competing financial interest. 


\section{ACKNOWLEDGMENTS}

This work was funded by KAUST (BAS/1/1346-01-01). The authors thank the KAUST Core Labs for technical assistance.

\section{REFERENCES}

(1) Dunn, B.; Kamath, H.; Tarascon, J. M. Electrical energy storage for the grid: a battery of choices. Science 2011, 334, 928-935.

(2) Choi, J. W.; Aurbach, D. Promise and reality of post-lithium-ion batteries with high energy densities. Nat. Rev. Mater. 2016, 1, 16013.

(3) Zhang, X.; Zhang, G.; Wang, S.; Li, S.; Jiao, S. Porous $\mathrm{CuO}$ microsphere architectures as high-performance cathode materials for aluminum-ion batteries. J. Mater. Chem. A 2018, 6, 3084-3090.

(4) Hwang, J.-Y.; Myung, S.-T.; Sun, Y.-K. Recent Progress in Rechargeable Potassium Batteries. Adv. Funct. Mater. 2018, 28, 1802938.

(5) Mao, M.; Gao, T.; Hou, S.; Wang, C. A critical review of cathodes for rechargeable $\mathrm{Mg}$ batteries. Chem. Soc. Rev. 2018, 47, 8804-8841.

(6) Ponrouch, A.; Frontera, C.; Bardé, F.; Palacín, M. R. Towards a calcium-based rechargeable battery. Nat. Mater. 2016, 15, 169-172.

(7) Elia, G. A.; Marquardt, K.; Hoeppner, K.; Fantini, S.; Lin, R.; Knipping, E.; Peters, W.; Drillet, J. F.; Passerini, S.; Hahn, R. An Overview and Future Perspectives of Aluminum Batteries. Adv. Mater. 2016, 28, 7564-7579.

(8) Lide, D. R. CRC Handbook of Chemistry and Physics; 90th Ed.; CRC Press, 2010.

(9) Lin, M. C.; Gong, M.; Lu, B.; Wu, Y.; Wang, D. Y.; Guan, M.; Angell, M.; Chen, C.; Yang, J.; Hwang, B. J.; Dai, H. An ultrafast rechargeable aluminium-ion battery. Nature 2015, 520, 325-328.

(10) Zhang, M.; Xiang, L.; Galluzzi, M.; Jiang, C.; Zhang, S.; Li, J.; Tang, Y. Uniform Distribution of Alloying/Dealloying Stress for High Structural Stability of an $\mathrm{Al}$ Anode in High-Areal-Density Lithium-Ion Batteries. Adv. Mater. 2019, 31, No. e1900826.

(11) Jiang, C.; Xiang, L.; Miao, S.; Shi, L.; Xie, D.; Yan, J.; Zheng, Z.; Zhang, X.; Tang, Y. Flexible Interface Design for Stress Regulation of a Silicon Anode toward Highly Stable Dual-Ion Batteries. Adv. Mater. 2020, 32, No. e1908470.

(12) Jiang, C.; Fang, Y.; Zhang, W.; Song, X.; Lang, J.; Shi, L.; Tang, Y. A Multi-Ion Strategy towards Rechargeable Sodium-Ion Full Batteries with High Working Voltage and Rate Capability. Angew. Chem., Int. Ed. Engl. 2018, 57, 16370-16374.

(13) Verma, V.; Kumar, S.; Manalastas, W., Jr.; Satish, R.; Srinivasan, M. Progress in Rechargeable Aqueous Zinc- and Aluminum-Ion Battery Electrodes: Challenges and Outlook. Adv. Sustainable Syst. 2019, 3, 1800111.

(14) Cohn, G.; Ma, L.; Archer, L. A. A novel non-aqueous aluminum sulfur battery. J. Power Sources 2015, 283, 416-422.

(15) Gao, T.; Li, X.; Wang, X.; Hu, J.; Han, F.; Fan, X.; Suo, L.; Pearse, A. J.; Lee, S. B.; Rubloff, G. W.; Gaskell, K. J.; Noked, M.; Wang, C. A Rechargeable $\mathrm{Al} / \mathrm{S}$ Battery with an Ionic-Liquid Electrolyte. Angew. Chem., Int. Ed. Engl. 2016, 55, 9898-9901.

(16) Yu, X.; Manthiram, A. Electrochemical Energy Storage with a Reversible Nonaqueous Room-Temperature Aluminum-Sulfur Chemistry. Adv. Energy Mater. 2017, 7, 1700561.

(17) Zhang, K.; Lee, T. H.; Cha, J. H.; Varma, R. S.; Choi, J.-W.; Jang, H. W.; Shokouhimehr, M. Two-dimensional boron nitride as a sulfur fixer for high performance rechargeable aluminum-sulfur batteries. Sci. Rep. 2019, 9, 13573.

(18) Zhang, K.; Lee, T. H.; Cha, J. H.; Jang, H. W.; Shokouhimehr, M.; Choi, J.-W. S@GO as a High-Performance Cathode Material for Rechargeable Aluminum-Ion Batteries. Electron. Mater. Lett. 2019, 15, $720-726$.

(19) Simoes, F. R. F.; Batra, N. M.; Warsama, B. H.; Canlas, C. G.; Patole, S.; Yapici, T. F.; Costa, P. M. F. J. Elemental Quantification and Residues Characterization of Wet Digested Certified and Commercial Carbon Materials. Anal. Chem. 2016, 88, 11783-11790.
(20) Smajic, J.; Alazmi, A.; Costa, P. M. F. J. The Role of the Binder/Solvent Pair on the Electrochemical Performance of Aluminium Batteries. MRS Adv. 2019, 807-812.

(21) Birch, M. E.; Ruda-Eberenz, T. A.; Chai, M.; Andrews, R.; Hatfield, R. L. Properties that influence the specific surface areas of carbon nanotubes and nanofibers. Ann. Occup. Hyg. 2013, 57, 11481166.

(22) Ward, A. T. Raman Spectroscopy of Sulfur Sulfur-Selenium and Sulfur-Arsenic Mixtures. J. Phys. Chem. 1968, 72, 4133.

(23) Smajic, J.; Alazmi, A.; Patole, S. P.; Costa, P. M. F. J. Singlewalled carbon nanotubes as stabilizing agents in red phosphorus Liion battery anodes. RSC Adv. 2017, 7, 39997-40004.

(24) Graupner, R. Raman spectroscopy of covalently functionalized single-wall carbon nanotubes. J. Raman Spectrosc. 2007, 38, 673-683.

(25) Helen, M.; Diemant, T.; Schindler, S.; Behm, R. J.; Danzer, M.; Kaiser, U.; Fichtner, M.; Anji Reddy, M. Insight into Sulfur Confined in Ultramicroporous Carbon. ACS Omega 2018, 3, 11290-11299.

(26) Pinkus, A. G.; Kim, J. S.; Mcatee, J. L., Jr.; Concilio, C. B. X-Ray Diffraction Pattern for Monoclinic Sulfur. J. Am. Chem. Soc. 1959, 81, $2652-2654$.

(27) Kumanek, B.; Janas, D. Thermal conductivity of carbon nanotube networks: a review. J. Mater. Sci. 2019, 54, 7397-7427.

(28) Yue, S. Y.; Ouyang, T.; Hu, M. Diameter Dependence of Lattice Thermal Conductivity of Single-Walled Carbon Nanotubes: Study from Ab Initio. Sci. Rep. 2015, 5, 15440.

(29) Fang, R.; Li, G.; Zhao, S.; Yin, L.; Du, K.; Hou, P.; Wang, S.; Cheng, H.-M.; Liu, C.; Li, F. Single-wall carbon nanotube network enabled ultrahigh sulfur-content electrodes for high-performance lithium-sulfur batteries. Nano Energy 2017, 42, 205-214.

(30) Simoes, F. R. F.; Batra, N. M.; Emwas, A. H.; Costa, P. M. F. J. Validation of alkaline oxidation as a pre-treatment method for elemental quantification in single-walled carbon nanotubes. Anal. Methods 2019, 11, 1884-1890.

(31) Labib, M. E.; Thomas III, J. H.; Embert, D. D. The Effect of Heat Treatment on Sulfur in an Electrically-Conductive CarbonBlack. Carbon 1984, 22, 445-451.

(32) Smajic, J.; Alazmi, A.; Batra, N.; Palanisamy, T.; Anjum, D. H.; Costa, P. Mesoporous Reduced Graphene Oxide as a High Capacity Cathode for Aluminum Batteries. Small 2018, 14, No. e1803584.

(33) Polo-Luque, M. L.; Simonet, B. M.; Valcárcel, M. Functionalization and dispersion of carbon nanotubes in ionic liquids. TrAC, Trends Anal. Chem. 2013, 47, 99-110.

(34) Imadate, K.; Hirahara, K. In Situ Observation of Wetting Ionic Liquid on a Carbon Nanotube. Langmuir 2016, 32, 2675-2678.

(35) Polo-Luque, M. L.; Simonet, B. M.; Valcárcel, M. Ionic liquid combined with carbon nanotubes: a soft material for the preconcentration of PAHs. Talanta 2013, 104, 169-172.

(36) Zhao, M.; Li, B. Q.; Peng, H. J.; Yuan, H.; Wei, J. Y.; Huang, J. Q. Lithium-Sulfur Batteries under Lean Electrolyte Conditions: Challenges and Opportunities. Angew. Chem., Int. Ed. Engl. 2020, 59, 17.

(37) Yang, H.; Yin, L.; Liang, J.; Sun, Z.; Wang, Y.; Li, H.; He, K.; Ma, L.; Peng, Z.; Qiu, S.; Sun, C.; Cheng, H. M.; Li, F. An AluminumSulfur Battery with a Fast Kinetic Response. Angew. Chem., Int. Ed. Engl. 2018, 57, 1898-1902.

(38) Nguyen, T. Q.; Breitkopf, C. Determination of Diffusion Coefficients Using Impedance Spectroscopy Data. J. Electrochem. Soc. 2018, 165, E826-E831.

(39) Deng, Z.; Zhang, Z.; Lai, Y.; Liu, J.; Li, J.; Liu, Y. Electrochemical Impedance Spectroscopy Study of a Lithium/Sulfur Battery: Modeling and Analysis of Capacity Fading. J. Electrochem. Soc. 2013, 160, A553-A558.

(40) Meng, X.; Cao, Y.; Libera, J. A.; Elam, J. W. Atomic Layer Deposition of Aluminum Sulfide: Growth Mechanism and Electrochemical Evaluation in Lithium-Ion Batteries. Chem. Mater. 2017, 29, 9043-9052.

(41) Yu, X.; Boyer, M. J.; Hwang, G. S.; Manthiram, A. RoomTemperature Aluminum-Sulfur Batteries with a Lithium-Ion-Mediated Ionic Liquid Electrolyte. Chem 2018, 4, 586-598. 
(42) Donohue, P. C. High-pressure spinel type Al2S3 and MnAl2S4. J. Solid State Chem. 1970, 2, 6-8.

(43) Yan, J.; Liu, X.; Li, B. Capacity Fade Analysis of Sulfur Cathodes in Lithium-Sulfur Batteries. Adv. Sci. 2016, 3, 1600101. 